\title{
The Design and Implementation of the Integrated Model of the Advertisement and Remote Control System for an Elevator
}

\author{
Woon-Yong $\mathrm{Kim}^{1}$ and SoonGohn $\mathrm{Kim}^{2 *}$ \\ ${ }^{1}$ Department of Computer \& Internet Technique, Gangwon Provincial College, 115 \\ Gyohang-ro, Jumunjin-eup, Gangneung-shi, Gangwon-do, 210-804, Korea \\ ${ }^{2}$ Department of Computer Science, Joongbu University, 101 Daehakro, Chubu-Meon, \\ GumsanGun, Chungnam, 312-702, Korea \\ 1'wykim@gw.ac.kr, ${ }^{2}$ sgkim@joongbu.ac

\begin{abstract}
Recently, There are various approach for an elevator system based on information technology. And an elevator that has more than 20,00 parts for the building and use by many and unspecified people publicity as a means of transporiation need to make the more secure and efficient management. For this purpose, the elevator systems require the more efficient network environment. But the service implementation of the network enabled all elevator is difficult because of the lack of IP. So in this paper, wC Cropose the methods of the configuration for the notification, advertisement and remote entrol of the elevators. This approach has inner network environment for the elevator to solve the lack of IP, and connect the management server with internet. And also, this configuration has connection of the smart phone. It can be notified about the problems of the elevator with cloud messaging method. And the advertisement service can be update $F$ and managed with remote unit easily. This approach can be supported the efficient and secure management.
\end{abstract}

Keywords: Remote Control, Advertisement, Elevator, Cloud Service, Emergency Call, Remote Management System, SmartPhone

\section{Introduction}

Recently, Ele ator accident is rising with spread of an elevator. And also it required various service environments for performance and crime enhancements. Recently, compulsory installation provisions of CCTV and direct call device was made for preventing an elevator accidents. And also there are various researches for improving the performance and maintenance environment. To deal with efficient management, the elevator systems require the more efficient network environment. But the service implementation of the network enabled all elevator is difficult because of the lack of IP. So in this paper, we propose the methods of the configuration for the notification, advertisement and remote control of the elevators. This approach has inner network environment for the elevator to solve the lack of IP, and connect the management server with internet. And also, this configuration has connection of the smart phone. It can be notified about the problems of the elevator with cloud messaging method. And the advertisement service can be updated and managed with remote unit easily. This control system optimizes a variety of communications environments anytime and has connection structure for emergency using cloud messaging service and the remote control system. This system which has the diagnostic capabilities support a field

* Corresponding Author 
engineer to make decision more quickly for reliable elevator environment to ensure the safety of passengers. Management of the current elevator is difficult to intensive monitoring and inspection because of a decrease in the maintenance of price and operating the hundreds unit per field engineers. Because of these situations, more effective management measures are required. For these problems, in this paper, we suggest the effective communication environment with management server and smart phone in order to control emergency situation. And the field engineer can recognize problem situations quickly and control the elevator remotely and directly.

The remaining parts of the paper are organized as follows, In Section 2, we discuss the related technology for the configuration, cloud and emergency service of the elevator system. In Section 3, we propose the system integrated model that is deal with configuration of the units around the elevator and architecture of the system. Section 4 presents operation and features of the proposed system and Section 5 concludes this paper.

\section{Related Works}

\subsection{Integrated Control Services of an Elevator System based on Information Technology}

In the operation of the elevator, the devices of the emergency control service such as CCTV, emergency bell and monitoring system are utilized in the elevator system mainly. The emergency bell is connected to a management office and a guardhouse directly. It can contact the manager when oceurring emergency situation by calling [1]. This situation has disadvantage that Grakes a lot time to solve the emergency situation when the manager is not exist. In addition, it is difficult accurate assessment and recognition about the situation of the problem because the caller delivers their situation and location orally [2] For solving of these problems, a different approach that the caller can connect other manager around any time is required when the manager absence [3]. In addition the monitoring system has lack of aggressive problem-solving skills because the mânagers identify the elevator situation manually. An important element of elevator accidents handling is securing safety and faster processing. For this purpose, the emergency control, system needs approaches to be diagnosed by the elevator specialists and to solye the problem in the fastest way.

Recently, with expansion of IT convergence technologies, the elevator service environment is rapidy changing. Mainly, the services have evolved to improvement of the user convenience and advancement services [4, 5]. And also there are various services such as notice and information service, elevator call and boarding service, elevator diagnostic and checking service so on $[6,7]$.

\subsection{Messaging in the Cloud}

Cloud computing is one of the hottest topics in the computer industry recently. And the cloud concept could change the messaging development process in the corporate IT in a very fundamental way. The cloud service provider would provide the cloud infrastructure and messaging service implementation. One of the typical cloud message services is Google Cloud Messaging for Android. This service allows users to send data from your server to the android device. This could be a lightweight message that can contain up to $4 \mathrm{~kb}$ of payload data telling the app there is new data to be fetched from the server. The follow picture (Figure 1.) shows the basic flow of cloud to device messaging. 


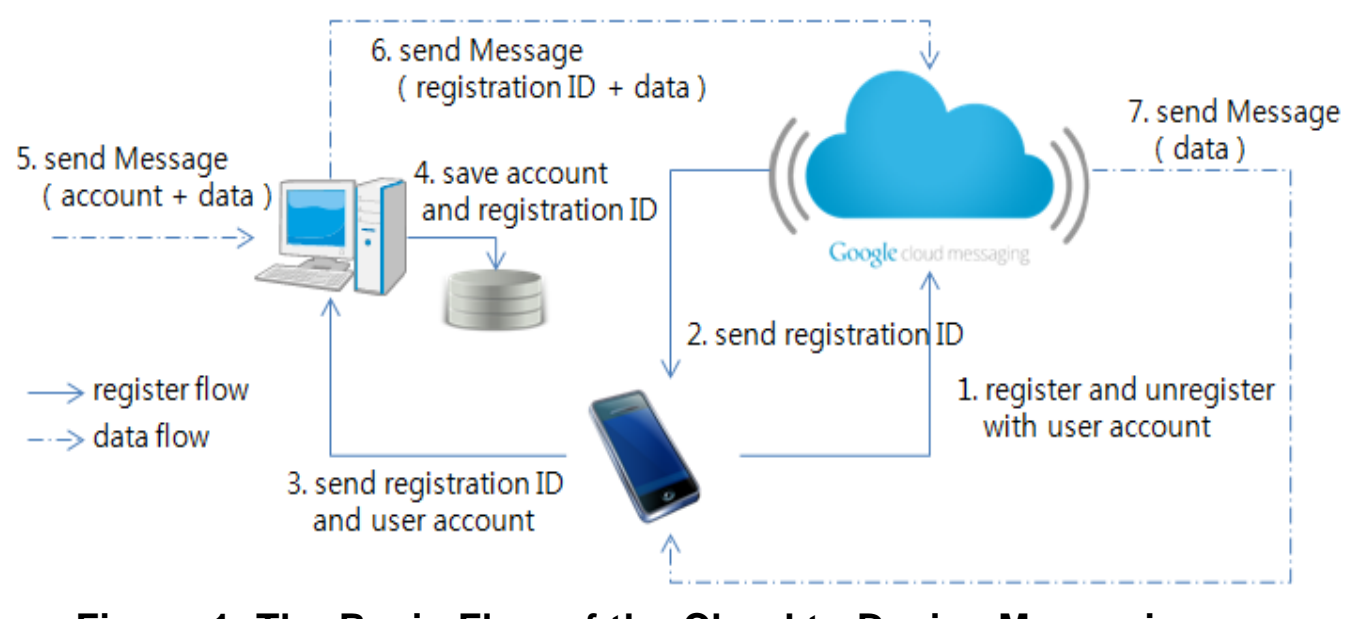

Figure 1. The Basic Flow of the Cloud to Device Messaging

The Cloud Messaging Service can be used to notify the situation of the emergency of the elevator. The proposed system use the cloud messaging service to optimize a variety of communications environments anytime and has connection structure for emergency using cloud messaging service and the remote control system. This system has the diagnostic capabilities to support a field engineer to make decision morequickly for reliable elevator environment to ensure the safety of passengers.

\section{The Integrated Model of the Elesator for Remote Control with Information Technology}

\subsection{The Proposed Service System Architecture}

The proposed integrated model of the elevator includes the ability to deal with various situations through the entire process of emergency among the manager, the field engineer and a maintenance company, and management of the advertisement system for the elevator This system establish communication environment that can be connected at any time and send elevator status at real time to the field engineer to solve the problems. This architecture has the configuration for the notification, advertisement and remote control of the e evators. This approach has inner network environment for the elevator to solve the lack of IP, and connect the management server with internet. And also, this configuration has connection of the smart phone .It can be notified about the problems of the elevator with cloud messaging method. And the advertisement service can be updated and managed with remote unit easily. The elements of the architecture is contron tower that is for the monitoring system in the management company of the elevator, the cloud server that can send the notification to the field engineer for emergency situation, smart phone application that can deal with the remote elevator system, web server and management server that can connect the remote management system in the elevator and manage the elevator system , CCTV, display unit, and remote management system connected to control panel of the elevator. The system architecture of the integrated model of the elevator control system is shown in Figure 2. 


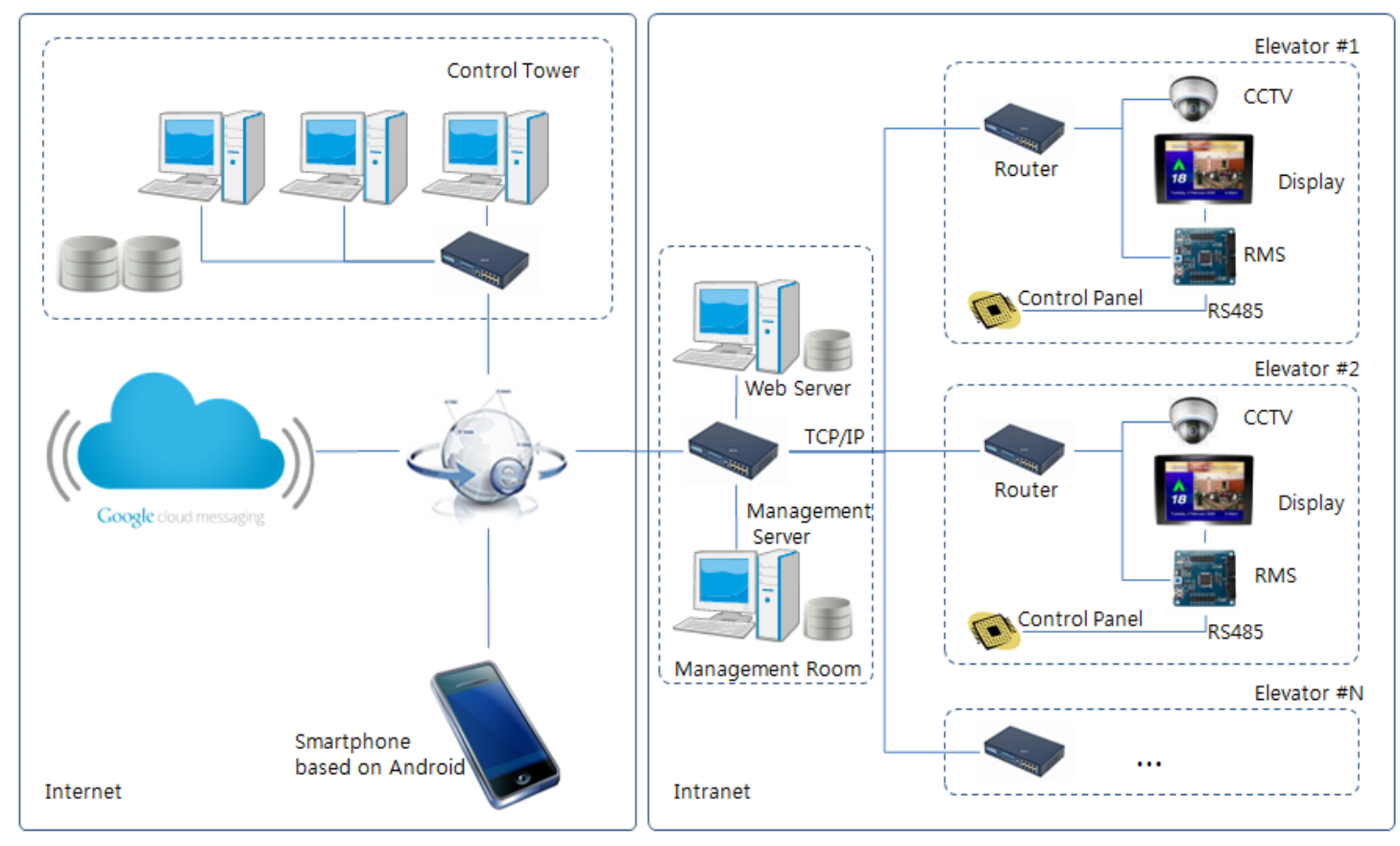

Figure 2. The System Architecture of the Integrated-Model of the Elevator Control Systen for an Elevator

The elevator system include of CCP and Advertisement Unit and RMS (Remote Management System) and operate with CP(Control Panel) organically. And also the elements are integrated into the RMS unit. The RMS control box sends and receives data of CCTV and Advertisement Unit and Control Panel of the elevator. And the management server in the management local room is connected with RMS control box. The server checks the RMS status and ensures connectivity of the elevator systems. And also the system acts as a remote control gateway. The manager in Control tower can connect to the management server in the management local room using internet line. And the smart phones cooperate with Google cloud server and are able to recognize the problem of the elevators in the remote location by sending the notification from cloud server. Smartphone-based remote control system provides a service that can recognize and respond elevator situation effectively with CCTV and RMS.

\subsection{The RMS Controlier Architecture}

The RMS Controller can control all status of the elevators, display units and management server. The RMS controller architecture is shown in Figure 3. In the system architecture (left), the system has Codec/Audio management and HDMI for display Advertisement service, communication module for analysis of the elevator control information based on RS232, RS485 and CAN, Memory and Connectivity module and Power management module, Connectivity module for LAN and USB with Process Unit. And also in the software architecture (right), the software is operated based on Linux and Android system [8] and have the various module such as communications and media process module and connectivity module. To communication for the control panel of the elevator, the system has various communication structures such as UART (RS232, RS485) and CAN to control devices of the elevators that made by various company. And JNI use to make the connection to android framework. RMS Service module consists of some module to need operations of management server, web server, control server and smart phone. We will present next parts. 

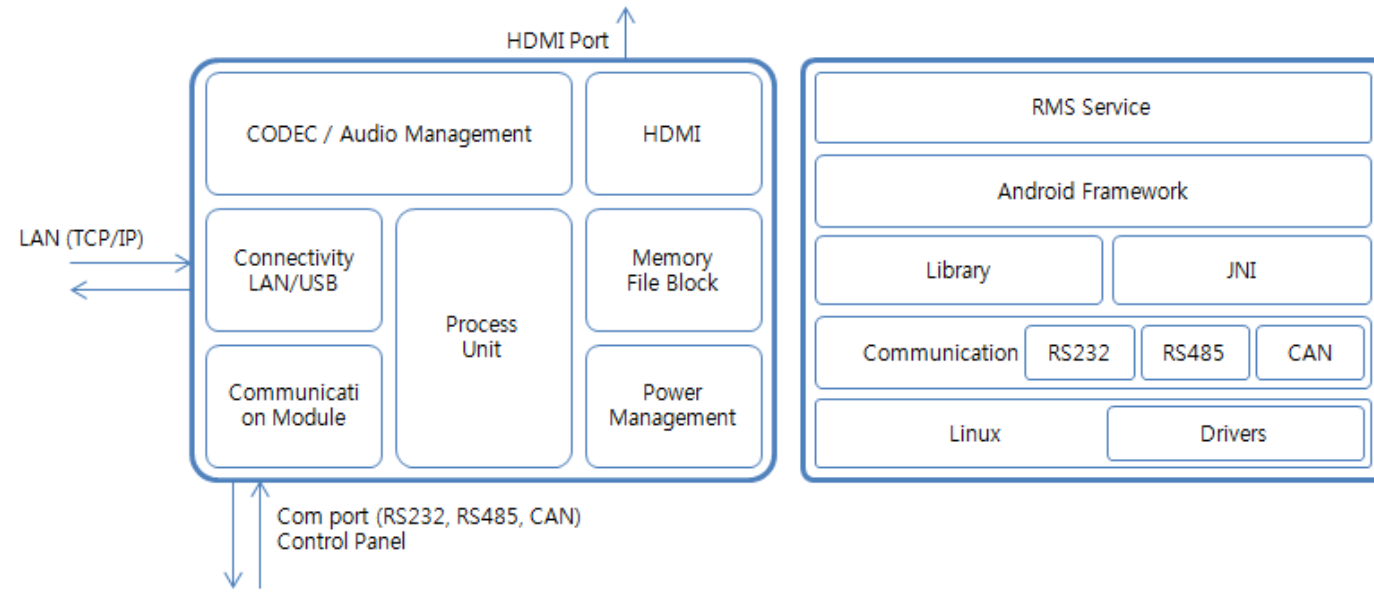

Figure 3. The RMS Control Box Hardware (Left) and Software (Bight) Architecture

\subsection{The RMS Service Module}

The RMS Service Module consists of 13 parts stich as Connector to the Control Panel, Connector to Internet Service, Database Manager, File, Manager, Device Control, Web Control, Notification Manager, Preference, Manager, Security Manager, RMS Status Manager and Advertisement Manager. The service module structure is shown in Figure 4.

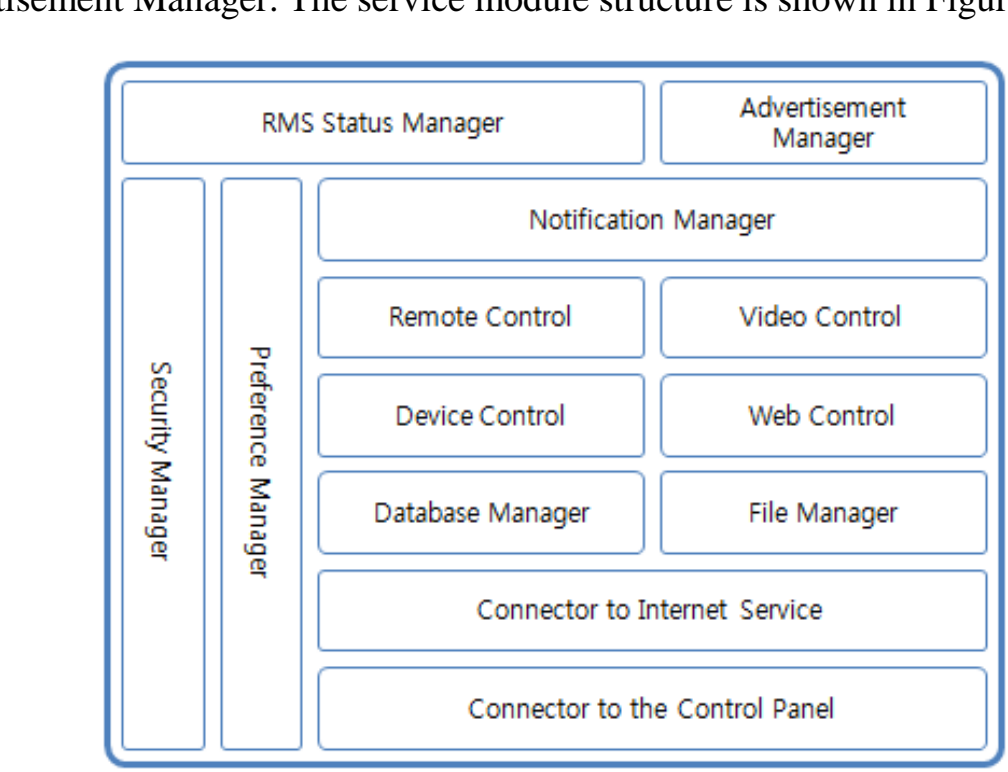

Figure 4. The Remote Management Service Module Structure

In the Connector to the Control Panel, the module controls to connect the Control Panel of the Elevator and has various communication protocols such as RS232, RS485 and CAN. It created by JNI. The module sends the received data from Control Panel to the Device Control module. In the Connector to Internet Service, this module maintains the status of the connection for the Management Server in the Management Office when the RMS started. In the Security Manager, It can control user permissions for making the structure available only authenticated users. In the Preference Manager, it manages a variety of environmental 
information such as the type of connection, location, permissions, and services methods and so on. In the Device Control, this module analysis the information received from the Connector to the Control Panel and the result are forward to the RMS Status Manager. If it received the abnormal signal, it also sends the abnormal signal to the Remote Control to deal with the situation immediately. Remote Control sends and receives data and commands from remote units. When the module received abnormal signal from Device Control, the module notify this situation to the Notification Manager for sending the data to remote filed engineers. And also when the module received commands from remote units, the module send the command to the Device Control Module to control devices. Notification Manager operate based on services. This module detects the abnormal signal from devices and the update signal from remote devices. When the module detects the abnormal signal form devices, it notifies the signal to the Management Server to know the situation to the managers. RMS Status Manager shows the current status of the elevator and can send command to control devices.

For the advertisement service, there are some modules such as File Manager, Wb Control, Video Control, Notification Manager and Advertisement Manager. In the File Manager, It control video files to save and sync data from the remote advertisement server. In the Web Control, It control web-based image and notice information from remote web server. It can recognize the update status from web server and eceiye data. In the Video Control, this module plays the video data using the video file that managed by File Manager module or play the video from on-line streaming service. Notification Manager checks the update status of the video and web-based contents. If ther exists the update status, it notifies the situation to the Video Control and Web Control model. In the Adyertisement Manager, this module display advertisement service such as notice, video web-based contents, weather and so on.

\section{The Operation of the Integrated Model for the Advertisement and Remote Control System of an Elevator}

\subsection{The Flows of the Remote Management Control System}

The Remote Control System has purpose to make a more safety environment and more efficiently services by providing seamless services, effective judgment from the situation of the elevators and a wealth of information to passengers. For this service implantation, we use several of devices to expand for providing a convenient and reliable service of information technology to the elevator systems. There are some devices such as CCTV, Emergency Call, Display Unit, Advertisement Server, Monitoring Server, Web Server, Mobile Unit and Control Panel of the Elevator so on. In this paper, we present the required equipment for the proposed service and show the relationship among them. And we present essential process to connect local network and internet environment, to notify the situation to the mobile unit and deal with advertisement service. First we present the flow of the network connection each element. Jhis flow is shown in Figure 5. 


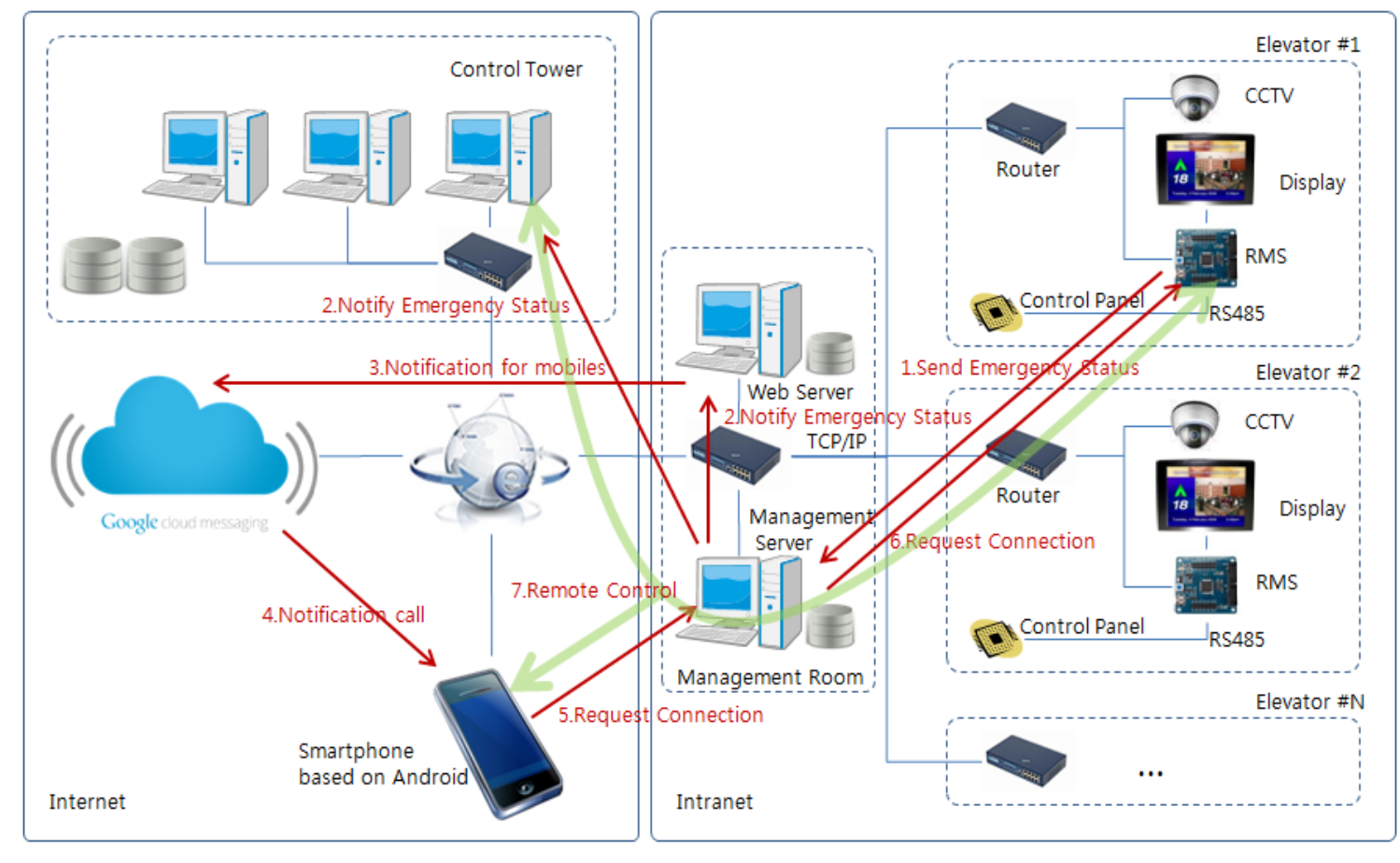

Figure 5. The Network Connection Flow of the Devices of the Elevator System

The Figure 5 shows the relationship ffoonnection and related devices when the elevator occur the abnormal signal. The cooperation model consist of RMS in the elevator, Display, CCTV, Management Server, Web Server, Goggle Cloud Messaging Server, Control Tower Server and Smartphone. The elevator system and Management Room have internal network environment. The all elevator system is Managed by Management Server and Web Server. When the elevator system lake an abnormal signal, the system notify to the RMS system. The RMS system sends the emergency status to the Management Server. When the Management Server received the emergency status, the server sends the status to the Web Server and Control Tower Server at the same time. In the Control Tower Server, this server request connection of the RMS to the Management Server. The Management Server makes the port of the RMS and notifies the Port information to the Control Tower Server. After the connection is compleled, they can send and receive the data from the RMS through the Management Server. And also The Management Server sends the emergency status to the Web Server to notify the status to the Smartphone. When the Web Server received the emergency stattus, this server searches the registered IDs for the Smartphone in the database. And then the server sends the registered IDs to the Google cloud messaging server. The Google cloud messaging server notifies the situation to the registered Smartphone. When the phone received the notification from Google cloud messaging server, the smart phone check the emergency status and then try to connect the RMS system through the Management Server. The Management Server will continue to maintain the connection with the RMS system. The relationship between the intranet of the elevator system that use the private IP and the internet network that use the public IP are maintained by the Management Server in the Management Room.

The Picture 6 shows the flow between the each system to make connection when the emergency status is occurred. 


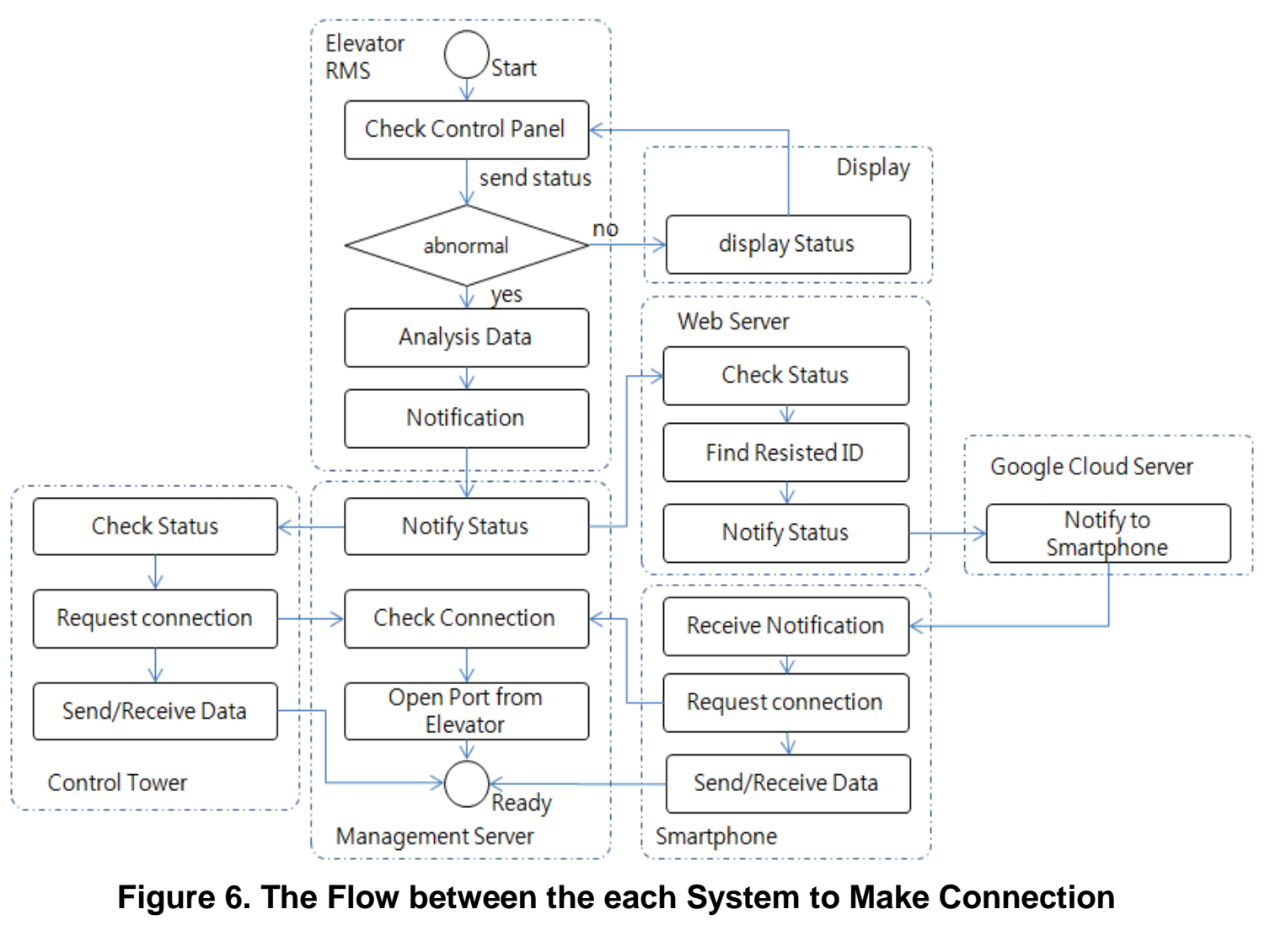

This flow start when the control panel checks the status of the elevator, the control panel sends data periodically. If the data is normal, show the data in the display panel and then listen another status. If the data is abnornal, the RMS analysis data and notify the notification information to the notification service, the notification service send the data management server. When the management server received notification, it sends the notification to the Control Tower and Web Server. The Control Tower Server requests the connection to the management server. When the connection completed, the Control Tower Server send and receive data to know and control the status. When the Web Server received the notification, it finds the registered ID form database and then the information sends the Google Cloud Messaging Server. The Mressaging notify the status to the Smart phone. The Smartphone try to connect to the Management Server that has a port to control the RMS system.

\subsection{Registration of the Devices in the Management Server}

The process of registration of the devices is made by the Management Server. The Management Server registers all device information such as all elevators in the building, RMS, User, Control Tower Server and Web Server. Before the installation of the RMS devices, The RMS device information has to register in the Management Server. The information is the Group Name, RMS ID, local network address and Managers so on. The registered RMS device can be control by the Management Server. And also the server has the information of the Web Server address and Control Tower Server address. The Web Server manages the registered ID and connections for the smart phone. 


\subsection{The Flow of the Advertisement Service}

The Advertisement Service is operated based on streaming data or video files with webbased contents. The flow shows in the Figure 7.

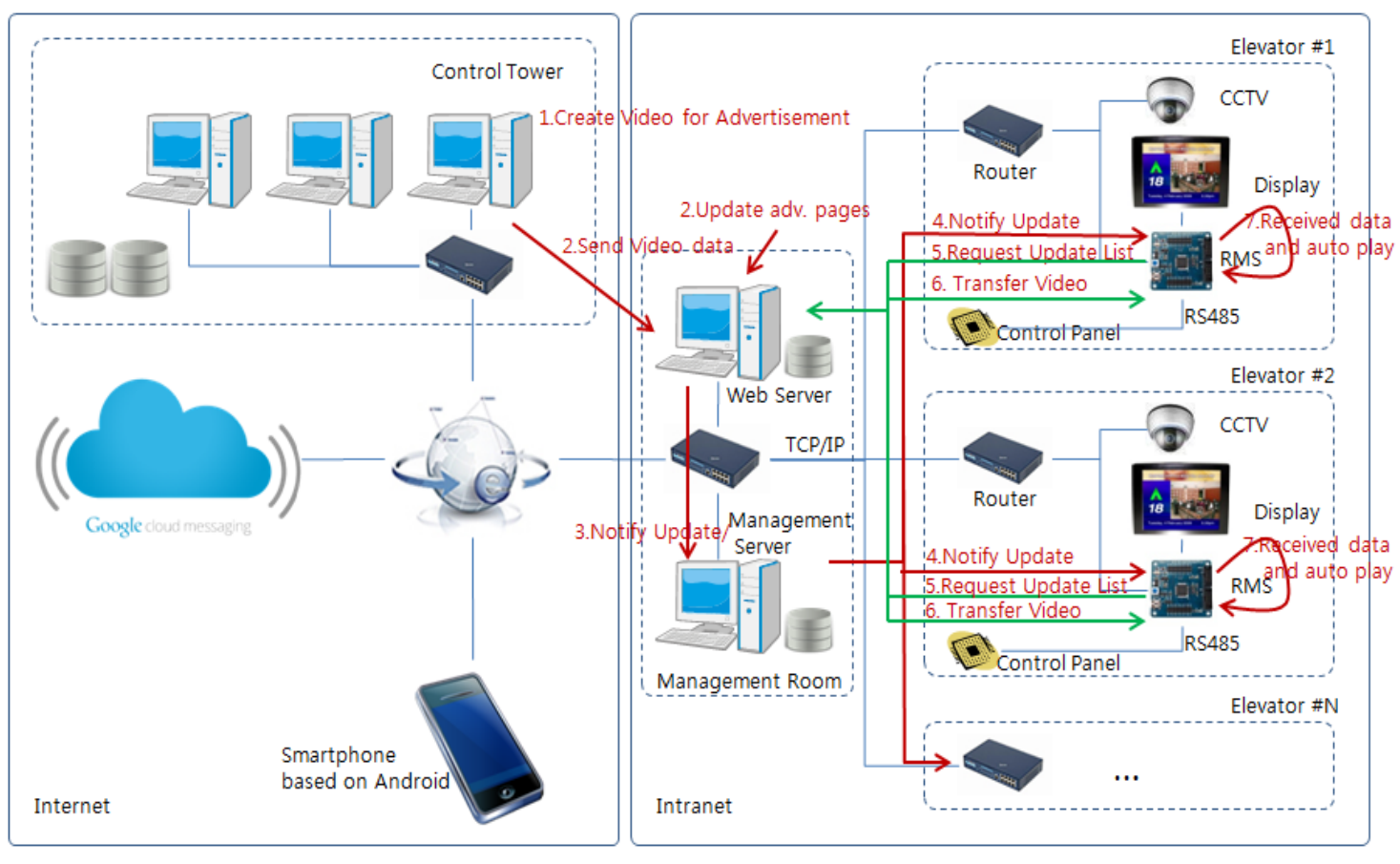

Figure 7. The Flow of the Ad yertisement Service based on Video Files and Web Contents

The flow shows the process of the advertisement data among the devices of the elevator. After the advertisement files are created, Control Tower Server sends the data to the Web Server. The Web Server can update advertisement contents such as video and web-based image contents. When the server received the request, the web server notifies the update requirement to the Managenent Server that control all devices of the elevators. The Management Server notifes update information to the RMS system of the all elevators. When the RMS received the potification, the RMS systems try to connect to the Web Server and request the update list of the advertisement and then the RMS system syncs all advertisement data from the Web Server. When the download finished, the RMS system play the advertisement contents to the Display unit automatically.

We wills show the flow of the relationship of the related device and services in the Figure 8. The flow descripts the relation of the Control Tower Server, Web Server, Management Server and RMS devices of the elevators. 


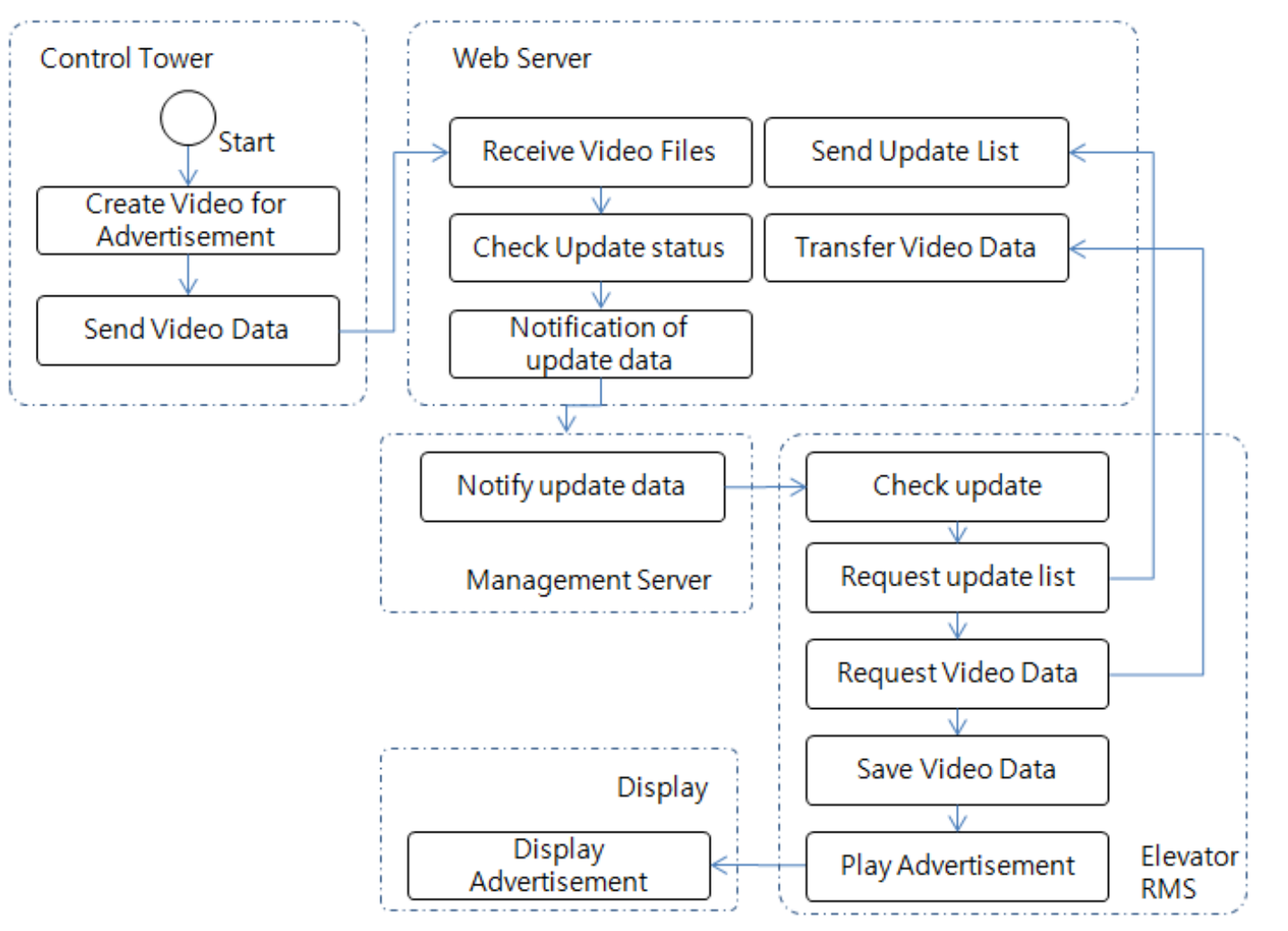

Figure 8. The Flow of the Relationship of the Related Device and Services

This figure shows the relationship of the Clated devices such as Control Tower, Web Server, Management Server, RMSs and Display unit in the elevator. Control Tower Server just sends the advertisement data to Web Server. And Web Server manages the contents of video, audio and images based on web contents. The server maintains the list of update and notifies the update situation to the Management Server, and sends the request contents to the RMS system. RMS maintains the advertisement data and syncs with the contents in the Web Server. And also the RMS plays the contents automatically when all the receiving data were completed.

\subsection{The Implementation Model of the Proposed System}

We show the mplementation model of the proposed system in the Figure 9. The model consists of 5 parts such as Control Tower, Smartphone, Web Server, Management Server and RMS system In the Control Tower, the system shows the status of the elevators and controls the elevator remotely. In the Web Server, the server manages the web contents for advertisement service and notifies the update information to the management server. And also this server manages registered IDs for smart phone to send notification of emergency situation. Management Server can register a various status for the elevator control such as user information, Control Tower and Web Server location, advertisement service methods such as streaming and file-based service and RMS information such as group ID, device ID, location so on. And also the Management Server maintains the connection of the RMS system and control remote control services. The RMS system in the elevator has the functions such as the advertisement service and elevator control service. The system connects the Control Panel of the elevator system and process the command of control for the elevator system. 


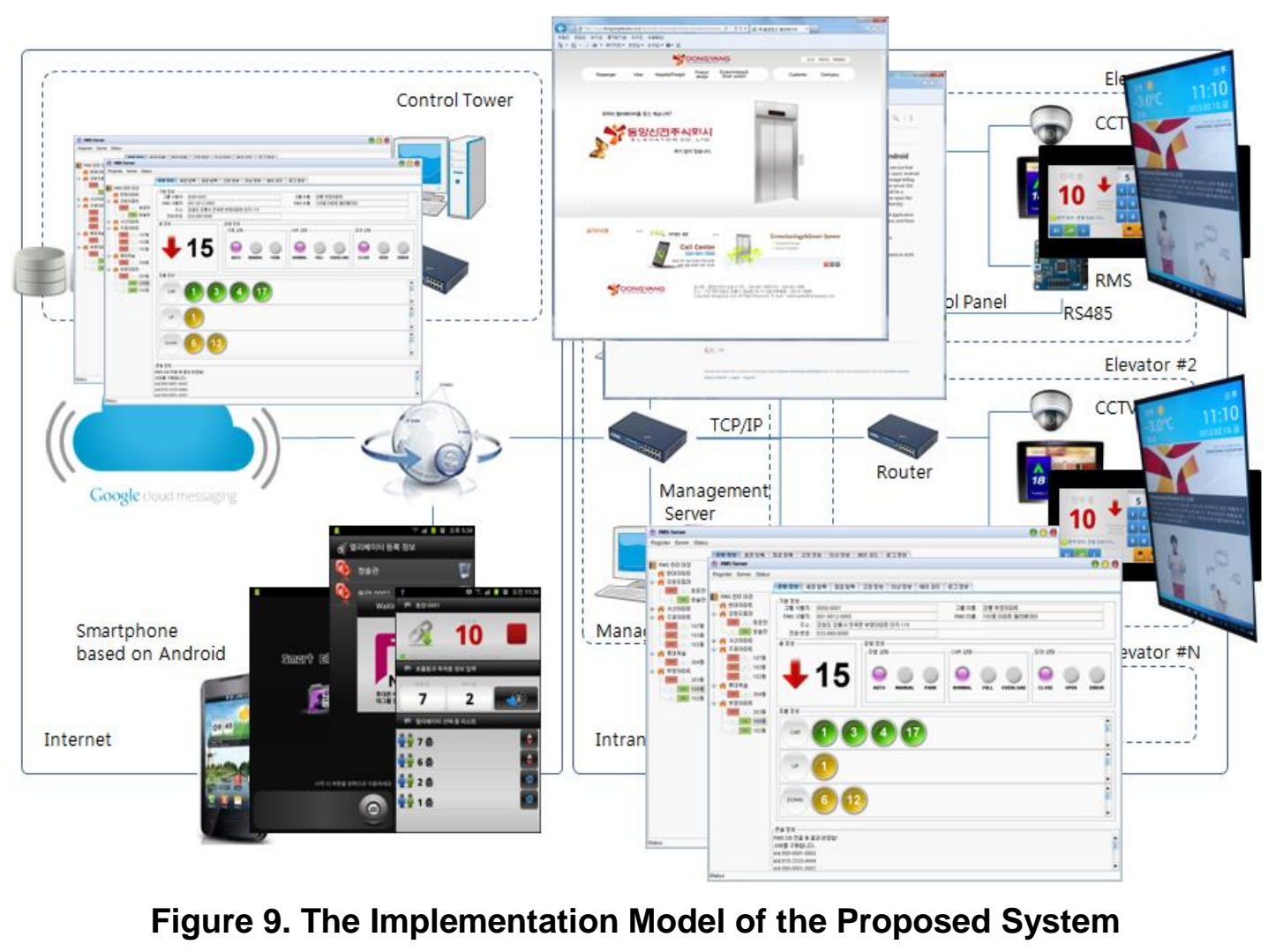

The system implements base on android system and java and tomcat. The Management Server and Control Tower Server were implemented as a java-based. And also the smart phone application and RMS system were implemented as an android system-based. And the communication module work based on the RS-485 communication.

\section{Conclusions}

With increasing demand and rapid change of the elevator industry, a variety of requirements is increasing for elevator safety awareness and efficient approach. So the elevator system required the more efficient network environment. But the implementation of the network enabled eleyator system is difficult because of the lack of IP. In this paper, we proposed the merhods of the configuration for the notification, advertisement and remote control of the elevators that has inner network environment for the elevator to solve the lack of IP and can remain connected status immediately and send the reason of the fault to the field enginee using the cloud messaging service so that the engineer can control the problem of the eleyator at real time remotely. And also we proposed the approach of the advertisement service. The proposed system will be responding to the passenger accidents quickly and will be able to build more reliable and secure environment of elevator maintenance.

\section{References}

[1] B. C. L. Yong-Hui Lee and H.-G. Kim, "A Study on Combined wire-wireless Service System for Emergency Warning Call”, Korea Information Processing Society Spring Conference, vol. 17, no. 1, (2010).

[2] S. Okazaki, "What is SMS advertising and why do multinationals adopt it? Answers from an empirical study in European markets", Journal of Business Research, vol. 61, no. 9, (2008). 
[3] A. Jotshi, "Dispatching and routing of emergency vehicles in disaster mitigation using data fusion", SOCIOECONOMIC Planning Sciences, vol. 43, no. 24, (2009).

[4] J. Jang, "Elevators of the latest technology trends", The Korean Society for elevator engineering, (2004) December.

[5] J-H. Jung, "A Study on the interface design of elevators in high rise building”, Hong-ik University, Doctoral Dissertation, (2007)

[6] H.-G. Seo and K.-H. Kim, "Design Methodology of a Real-Time Elevator Monitor and Control System", Journal of the Institute of Industrial Technology, vol. 26, no. 2, (1998) December.

[7] Y.-S. Choi, B.-T. Park and Y.-J. Choi, "Design and implementation of Location Based Silver Town uService System", Journal of Korean Society for Internet Information, vol. 11, no. 3, (2010).

[8] Google Android, http://www.android.com.

\section{Authors}

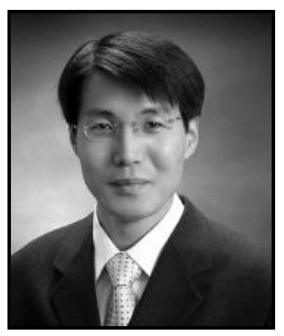

Woon-Yong Kim, he received Ph.D. degrees from Kwangwoon University, Seoul, Korea, in Computer Science in 2003. He has been working as an Assistant Professor in Gangwon Provincial College from March 2006. His research interests include Distributed Computing, Web Services, Embedded Software, A methodology of Software Development, Networks and so on

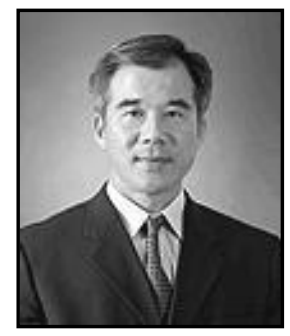

SoonGohn Kim, de received $P(D$, degrees from Chonbuk National University, Seoul Korea, in Conputer Engineering in 1999. He has been working as an Professor in Joongbu University from March 1995. His research interests Include Ubiquitous Computing, Distributed Computing, Database Integrity, Cryptographic Protocol, A methodology of Software Developnent, Software Evaluation, Networks and so on.
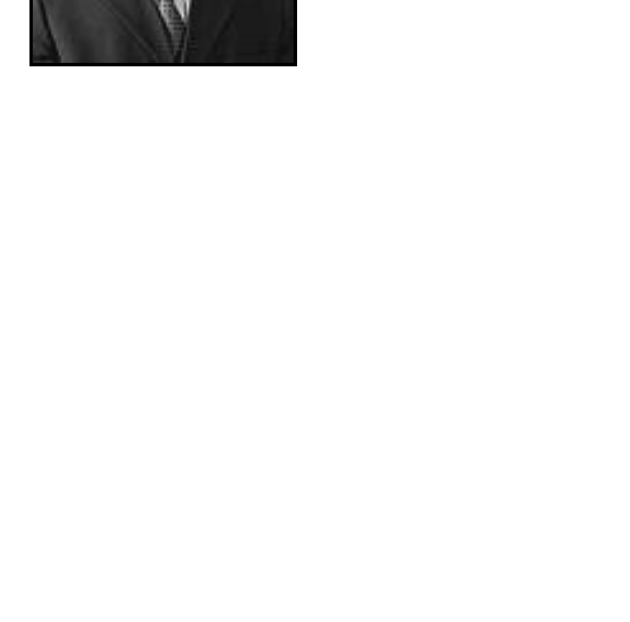